\title{
Impacto de los programas de bienestar universitario en la calidad de vida de los estudiantes
}

\author{
Alexander Daza-Corredor ${ }^{1}$ \\ María Paola Jiménez Villamizar ${ }^{2}$ \\ Farid Leonardo Rodríguez Pacheco ${ }^{3}$
}

Recibido: 04-03-2020

Aceptado: 06-05-2020

\section{Resumen}

El presente estudio tiene por objetivo identificar y describir el impacto de los programas de bienestar en la calidad de vida de estudiantes universitarios. Se realizó un estudio bibliométrico retrospectivo y descriptivo; se revisaron artículos indexados en la base de datos Scopus, de los cuales se seleccionaron 11 publicaciones para elaborar el presente artículo, teniendo en cuenta el título, afiliación de los autores, resumen y conclusiones.

1. Doctor en Ciencias Gerenciales. Docente de planta de la Universidad del Magdalena, Santa Marta, Colombia. Director del Grupo de Investigación en Gestión de las Organizaciones (GIGO).

Correo electrónico: alexdaza71@hotmail.com

ORCID: https://orcid.org/0000-0001-9792-0003

Google Scholar: https://scholar.google.com/citations?user=PzHTp 1 UAAAAJ\&hl=en

Cvlac: https://scienti.minciencias.gov.co/cvlac/visualizador/generarCurriculoCv.

do?cod_rh=0000331945

2. Magíster en Intervención Social en las Sociedades del Conocimiento. Docente catedrática de la Universidad del Magdalena, Santa Marta, Colombia, Grupo de Investigación Psicología de la Salud y Psiquiatría.

Correo electrónico: paolajimenez_8@hotmail.com

ORCID: https://orcid.org/0000-0003-2264-7422

Google Scholar: https://scholar.google.com/citations?user=gEaLutcAAAAJ\&hl=en\&oi=ao

Cvlac: https://scienti.minciencias.gov.co/cvlac/visualizador/generarCurriculoCv.do?cod_ $\mathrm{rh}=0001414191$

3. Magíster en Planificación Territorial y Gestión Ambiental. Integrante del Grupo de Investigación en Gestión Pedagógica Transformadora (GEPET), Universidad del Magdalena, Santa Marta, Colombia.

Correo electrónico: frodriguezum@gmail.com

ORCID: https://orcid.org/0000-0003-2176-1856

Google Scholar: https://scholar.google.com/citations?hl=en\&user=nhON_SMAAAAJ

Cvlac: https://scienti.minciencias.gov.co/cvlac/visualizador/generarCurriculoCv.

do?cod_rh $=0000017190$ 
El $81,8 \%$ de los estudiantes que participaron en algún programa de bienestar universitario tuvieron un impacto positivo en su calidad de vida. En un 18,2 \% no se hallaron diferencias significativas entre estudiantes que participaron y aquellos que no participaron. La calidad de vida es una variable transversal en los programas de bienestar universitario, lo que hace necesario evaluar otros factores al momento de establecer una estrategia de intervención.

Palabras clave: calidad de vida, programas de bienestar, estudiantes, universidad, revisión sistemática.

\section{Impact of university wellness programs on the quality of life of students}

\section{Abstract}

The objective of this study is to identify and describe the impact of wellness programs on the quality of life of university students. A retrospective and descriptive bibliometric study was performed; Articles indexed in the Scopus database were reviewed, of which 11 publications were selected to prepare this article, taking into account the title, affiliation of the authors, abstract and conclusions. $81.8 \%$ of the students who participated in a university wellness program had a positive impact on their quality of life. In $18.2 \%$, no significant differences were found between students who participated and those who did not participate. Quality of life is a cross-sectional variable in university wellness programs, which makes it necessary to evaluate other factors when establishing an intervention strategy.

Keywords: quality of life, wellness programs, students, university, systematic review. 


\section{Introducción}

La misión principal de las instituciones de educación superior (IES) es formar profesionales integrales, procurando brindar un ambiente de salud y bienestar. La sociedad demanda un servicio educativo de alta calidad, lo cual obliga a las universidades a diseñar acciones estratégicas orientadas a su mejora continua (Cáceres, Gamboa y Velasco, 2018). Así dejan de ser solo un centro de enseñanza, para convertirse en instituciones polifacéticas y multifuncionales, generadoras de conocimiento y prestadoras de servicios (Contecha y Jaramillo, 2011). Por estas razones los programas de bienestar universitario tienen un papel importante en la formación integral del estudiante, pues prestan un servicio que les permite ajustarse al medio en que se encuentran, para que desarrollen habilidades y competencias en la sociedad y que mejoren su calidad de vida (Pezzano, 2009).

La mejora de la calidad de vida requiere bienestar económi$\mathrm{co}$, tecnológico y material, procesos específicos que contribuyen al avance de la sociedad. El concepto de calidad de vida demanda un abordaje multidimensional, pues en él confluyen la valoración perceptiva del individuo sobre su satisfacción y bienestar en diferentes aspectos de su vida, por lo cual puede haber oscilaciones en esas percepciones (Pacheco, Michelena, Mora y Miranda, 2014).

Además de ser un índice de bienestar y satisfacción, el concepto de calidad de vida es considerado como un criterio importante para ver el impacto de un programa universitario (Bernal y Rivera, 2011). Es usado en las instituciones de educación superior para contribuir a mejorar los índices de bienestar relacionados con los estudios, pues está relacionado con la sensación de autoeficacia, satisfacción, compromiso e identidad institucional (Salanova, Martínez, Bresó, Llorens y Grau, 2005).

Cortés (2015) expone que la naturaleza del bienestar está relacionada con lo social. Los resultados de un estudio realizado por Estupiñán y Vela (2012) demuestran que la calidad de vida en las 
estudiantes universitarias que son madres jóvenes está relacionada con el estado de las relaciones familiares y la percepción del apoyo social recibido en el contexto educativo. Asimismo, su productividad, expresión emocional, salud y seguridad se fortalecen porque dependen de la aceptación de la maternidad en su contexto familiar y la institución de educación superior a la cual pertenece. Esto significa que la calidad de vida no se reduce a un entorno clínico, sino que, al ser un constructo multidimensional, requiere de estrategias para mejorar su calidad de vida en diferentes escenarios. Como las universidades son espacios donde existe una gran interacción, los niveles de bienestar y satisfacción deben mejorar para complementar la formación de profesionales integrales.

El concepto de calidad de vida está muy relacionado con la satisfacción de las necesidades y constituye un objetivo inicial e histórico de los programas de bienestar universitario (González, Aguilar y Pezzano, 2002). Rojas (2019) encontró que los estudiantes perciben que no todos los servicios ofertados por la dependencia de bienestar universitario influyen en la calidad del estudiante, pues encontró que el comedor es el mejor servicio en comparación con otros porque disponer de una alimentación adecuada aporta de manera significativa a sus vidas y reduce costos económicos.

El Ministerio de Educación Nacional (MEN) y las instituciones de educación superior (IES) deben fortalecer las políticas de bienestar universitario porque todos los esfuerzos en este sentido disminuirán significativamente la deserción estudiantil (MEN, 2009, p. 110). Por estas razones el presente estudio tiene por objetivo identificar y describir el impacto de los programas de bienestar en la calidad de vida de estudiantes universitarios, a través de una revisión sistémica.

\section{Materiales y métodos}

Para esta investigación se realizó un estudio bibliométrico retrospectivo y descriptivo (Ávila y Marenco, 2016), que se llevó a cabo 
en dos fases: una de búsqueda y otra de análisis. La fase de búsqueda consistió en la revisión de artículos indexados en la base de datos Scopus, utilizando los siguientes términos: (wellness AND programs) AND (university AND students) AND (quality AND of AND life), (programas AND bienestar) AND (universitarios) AND (calidad AND de AND vida).

Los criterios de elegibilidad de los artículos se basaron en las siguientes características: 1) artículos de investigación original, debido a que estos muestran de manera detallada la metodología científica, se descartaron artículos de revisión y reflexión; 2) términos de búsqueda en título, resumen o palabras claves; 3 ) participantes que son estudiantes universitarios, 4) idiomas español o inglés; 5) realizados durante los últimos 20 años (2000 a 2019).

Para la fase de análisis de los datos se emplearon los programas Microsoft Excel 2016 y SPSS versión 25. Para la depuración y selección de las 11 publicaciones para elaborar este artículo se tuvo en cuenta el título, afiliación de los autores, resumen y conclusiones.

\section{Resultados y discusión}

En la búsqueda se identificaron 299 artículos, de los cuales se excluyeron 239 porque no cumplían con los criterios de elegibilidad. Una vez realizada la lectura de los 60 artículos utilizados en la fase inicial se descartaron 49 que no cumplían con el objetivo de la investigación, como se observa en la figura 1 . 
Figura 1. Flujograma de proceso de selección de artículos estudiados.

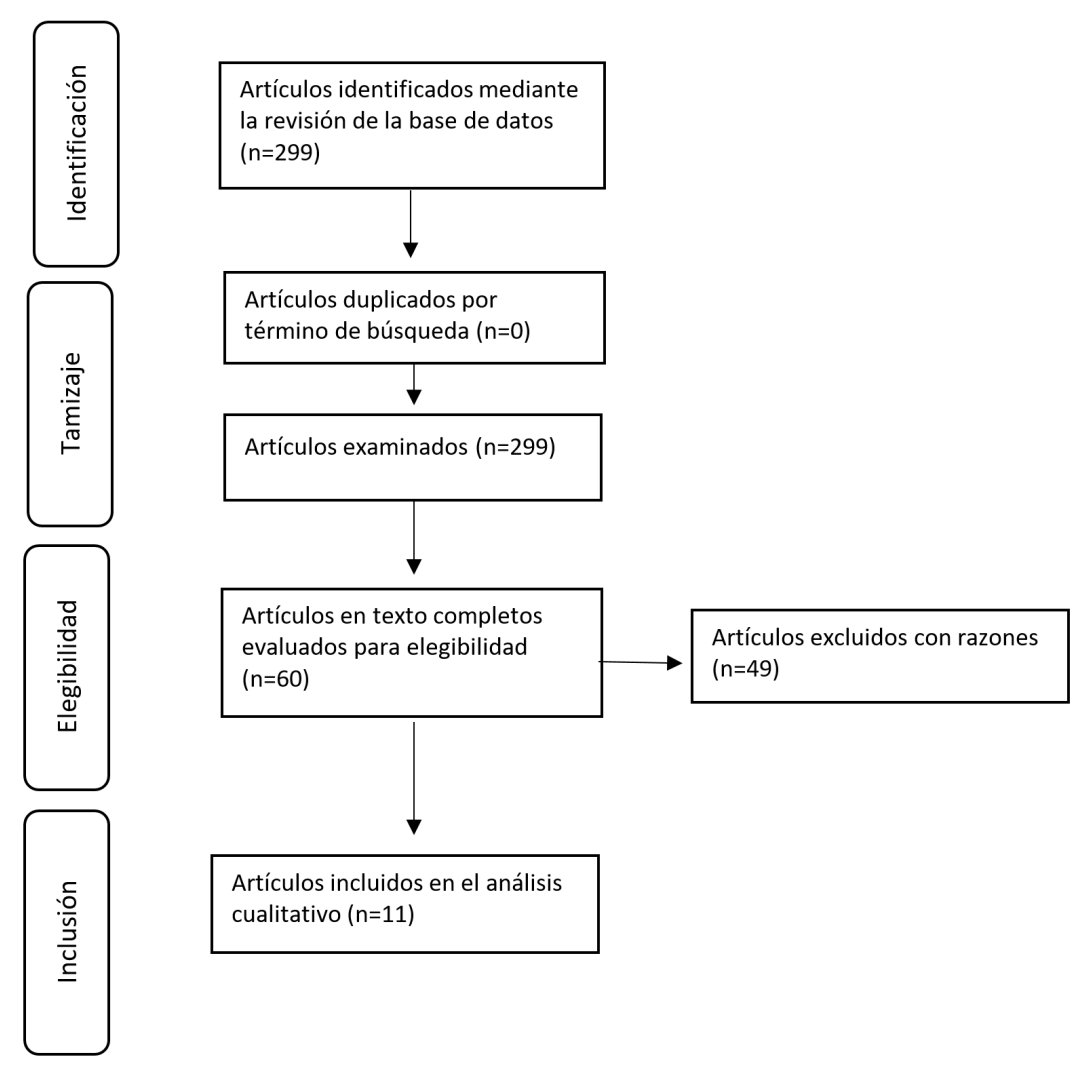

Fuente: Elaboración propia.

Los artículos analizados fueron publicados en los últimos 10 años (2010-2019), de estos el 36,3\% fueron publicados entre el 2015 y 2016, un $27,2 \%$ en el 2012. El 63,6 \% fueron investigaciones adelantadas en estudiantes universitarios estadounidenses, el 18 $\%$ en estudiantes españoles, el $9 \%$ de origen indonesio y el otro $9 \%$ colombianos. De las investigaciones analizadas el $81,8 \%$ de los estudiantes que participaron en algún programa universitario tuvieron un impacto positivo en su calidad de vida, en un $18,2 \%$ no hallaron diferencias significativas entre estudiantes que participaron y aquellos que no participaron. 
En estudiantes universitarios de Indonesia (Wahyun, Nurihsan y Yusuf, 2019) y Estados Unidos (McGrady, Brennan, Lynch y Whearty, 2012; Danitz y Orsillo, 2014) se implementaron programas de aceptación y compromiso para mejorar el bienestar y reducir síntomas depresivos. Se halló que entre mayor sea la aceptación, menores serán las variables que afectarán su percepción de bienestar e interferirán en su calidad de vida. A través de la implementación de un programa de aprendizaje en línea, D'Abundo, Marinaro y Fiala (2010) encontraron que hubo una reducción del consumo de cigarrillo en fumadores y la prevención del consumo en los no fumadores. Real, Zackoff, Davidson y Yakes (2015) sustentan que la mayor participación en un programa de bienestar no está asociada con la disminución del agotamiento, pero que la percepción que tiene el estudiante sobre la utilidad del programa sí disminuye su agotamiento mejorando así su bienestar y calidad de vida. Estos resultados pueden ser contrastados con el estudio de Salcedo, Quezada, Novillo, Varela, Núñez y Viteri (2017), realizado entre estudiantes de las universidades de la provincia de El Oro, en Ecuador, quienes consideraron que las unidades de bienestar estudiantil junto con sus programas permiten el desarrollo de las capacidades individuales y colectivas de toda la comunidad universitaria. Por su parte, Bonilla y Padilla (2015) implementaron estrategias meditativas para el manejo de la ansiedad (EMMA) en universitarios en Puerto Rico, y encontraron que esta intervención es viable y eficaz, pues aumentó su capacidad, tuvo efecto positivo y mejoró algunas dimensiones de su bienestar psicológico y de su calidad de vida(Gómez, Ostos, Gallego, \& García, 2019).

Algunas investigaciones sobre estudiantes universitarios de especialidades médicas en calidad de residentes encontraron que aquellos que participan de programas de bienestar mostraron mejores condiciones de bienestar, calidad de vida y salud mental en comparación con aquellos que no (Saadat, Snow, Ottenheimer, Dai y Kain, 2012; Runyan, Savageau, Potts y Weinreb, 2016). Lo mismo sucedió con investigaciones sobre estudiantes de maestría, en donde se evaluó la efectividad de un modelo de supervisión de bienestar y se observó un aumento en bienestar personal y total (Lenz, Sangganjanavanich, Balkin, Oliver y Smith, 2012). 
Por su parte, en investigaciones adelantadas con población de estudiantes universitarios adultos-mayores de 55 años, aquellos que se encuentran satisfechos con sus programas universitarios tienden a sentirse más útiles y consideran que el participar en esto les "refuerzan la autoestima" y les da "alegría de vivir y aprender", mostrando un impacto positivo en el bienestar y calidad de vida de los participantes (Fernández, García y Pérez, 2016). Por otro lado, en otros estudios realizados en estudiantes adultos-mayores no difirió de forma estadísticamente significativa la calidad de vida, apoyo social e inteligencia emocional entre aquellos que participaran o no en un programa de intervención (Lucas, Pérez, Fonseca y Ortuño, 2015; Fonseca, Pérez, Ortuño y Lucas, 2017). Estos datos se pueden contrastar con los hallados por Vilaplana (2010), quien encontró que participar en un programa universitario para mayores aumenta significativamente la probabilidad de que el individuo goce de una muy buena calidad de vida $(P=1 ; 0,5670)$. Esto difiere de lo expuesto por Gañán y Villafruela (2015), quienes dicen que el mejoramiento de la calidad de vida en los alumnos mayores no se debe a un factor único, sin embargo, es importante resaltar que identificaron que el asistir y permanecer en programas universitarios tiene una relación con la mejora de la calidad de vida y promoción de actitudes de envejecimiento activo.

\section{Conclusiones}

Los programas de bienestar tienen una gran importancia en la comunidad universitaria, en especial para el mejoramiento de la calidad de vida de cada miembro que la compone.

Los estudios con un encuadre experimental permiten hacer futuras investigaciones para evaluar la efectividad de los programas de bienestar. Los diseños experimentales presentan importantes ventajas para determinar los efectos de una intervención sobre una población específica. De esa manera es posible conocer si los objetivos del programa se están logrando realmente y cuál es el grado de cumplimento. 
Los resultados aquí encontrados nos permiten resaltar que la calidad de vida es una variable transversal en los programas de bienestar universitario, lo que hace necesario evaluar otros factores al momento de establecer una estrategia de intervención. Entendiendo la calidad de vida como un constructo multidimensional, esta requiere que cada entorno donde se desenvuelve el individuo desarrolle estrategias que permitan mejorar su calidad de vida. Como las universidades son espacios donde existe una gran interacción, mejorar los niveles de bienestar y satisfacción debe ser parte de sus metas para la formación de profesionales integrales.

La creación de las dependencias de bienestar universitario permite reafirmar el compromiso de las universidades con la formación integral de toda la comunidad académica (estudiantes; administrativos; docentes de planta, ocasionales, catedráticos; contratistas). Esas dependencias están deben estar orientadas no solo hacia la promoción y oferta de programas y servicios socioeconómicos en estudiantes (apoyos económicos, servicios de alimentación, etc.), sino también al fortalecimiento de las capacidades individuales, mediante servicios como la motivación académica, técnicas de estudio, fortalecimiento de habilidades cognitivas y emocionales, y otros que pueden ser mejor explicados desde disciplinas psicosociales. Esta oferta no solo mejora la calidad de vida, sino que promueve la permanencia, el rendimiento académico y la graduación de los estudiantes universitarios (Ostos-Ortiz \& Aparicio-Gómez).

\section{Referencias bibliográficas}

Ávila, J. y Marenco, A. (2016). Producción bibliométrica y redes de cooperación en la revista Psicología desde el Caribe. Psicología desde el Caribe, 33(1), 66-80. Recuperado de http://dx.doi.org/10.14482/ psdc.33.1.8063

Bernal, H. y Rivera, B. (2011). Responsabilidad social universitaria: aportes para el análisis de un concepto. Asociación Colombiana de Universidades, 21(2), 7-32. Recuperado de https://ascun.org.co/ 
uploads/default/publications/196b8df9ad25320abe0eceb1a0329879. pdf

Bonilla, K. y Padilla, Y. (2015). Estudio piloto de un modelo grupal de meditación de atención plena (mindfulness) de manejo de la ansiedad para estudiantes universitarios en Puerto Rico. Revista Puertorriqueña de Psicología, 26(1), 72-87. Recuperado de https://www.redalyc.org/ pdf/2332/233245620006.pdf

Cáceres, M., Gamboa, E. y Velasco, S. (2018). Satisfacción de estudiantes universitarios frente a un servicio de alimentación institucional y sus factores asociados. Nutrición Clínica y Dietética Hospitalaria, 38(3), 93103. Recuperado de http://dx.doi.org/10.12873/383caceres

Contecha, L. y Jaramillo, L. (2011). El bienestar universitario subordinado a una modernidad instrumental. Revista U. D. C. A. Actualidad \& Divulgación Científica, 14(1), 101-109. Recuperado de https://doi. org/10.31910/rudca.v14.n1.2011.762

Cortés, F. (2015). La ruta social de la razón en la universidad. Democracia y deliberación. Eidos, 23, 13-36. Recuperado de http://dx.doi. org/10.14482/eidos.23.184

D’Abundo, M. L., Marinaro, L. M. y Fiala, K. A. (2010). An online learning module focused on smoking education and prevention for college students: implications for college health instructors and allied health professionals. Journal of Allied Health, 39(1), 43-48. Recuperado de https://www.ncbi.nlm.nih.gov/pubmed/20217006

Danitz, S. y Orsillo, S. (2014). The mindful way through the semester: an investigation of the effectiveness of an acceptance-based behavioral therapy program on psychological wellness in first-year students. Behavior Modification, 38(4), 549-566. Recuperado de https://doi. org/10.1177/0145445513520218

Estupiñán, M. y Vela, D. (2012). Calidad de vida de madres adolescentes estudiantes universitarias. Revista Colombiana de Psiquiatría, 41(3), 536549. Recuperado de https://doi.org/10.1016/S0034-7450(14)60027-5 
Fernández, A., García, J. L. y Pérez, G. (2015). Grado de satisfacción de los adultos con los programas universitarios. Revista Complutense de Educación, 27(3), 1021-1040. Recuperado de https://doi.org/10.5209/ rev_RCED.2016.v27.n3.47239

Fonseca, E., Pérez, A., Ortuño, J. y Lucas, B. (2017). Efectos de una intervención de educación emocional en alumnos de un programa universitario para mayores. Universitas Psychologica, 16(1), 1-11. Recuperado de http://dx.doi.org/10.11144/Javeriana.upsy16-1.eiee

Gañan, Á. y Villafruela, I. (2015). El ocio, tiempo libre y calidad de vida para un envejecimiento activo, el caso de la Universidad de Burgos. EJIHPE: European Journal of Investigation in Health, Psychology and Education, 5(1), 75-87. Recuperado de https://doi.org/10.30552/ejihpe. v5i1.91

Gómez, O. Y. A., Ostos, O. L., Gallego, M. A. C., \& García, C. A. (2019). Análisis sistemático de los artículos publicados en la revista interamericana de educación, investigación y pedagogía (RIIEP) durante 2014 a 2019. Revista Interamericana de Investigación, Educación y Pedagogía, RIIEP, 12(2), 225-240.

González, L., Aguilar, E. y Pezzano, G. (2002). Reflexiones sobre bienestar universitario. Una mirada desde la educación a distancia y jornada nocturna. Bogotá: Instituto Colombiano para el Fomento de la Educación Superior. Recuperado de http://www.fumc.edu.co/wp-content/uploads/ resoluciones/arc_913.pdf

Lenz, A., Sangganjanavanich, V., Balkin, R., Oliver, M. y Smith, R. (2012). Wellness model of supervision: a comparative analysis. Counselor Education and Supervision, 51(3), 207-221. Recuperado de https://doi. org/10.1002/j.1556-6978.2012.00015.x

Lucas, B., Pérez, A., Fonseca, E. y Ortuño, J. (2015). Programas educativos universitarios para mayores: evaluación de su impacto en la autopercepción del apoyo social y la salud mental. Revista Colombiana de Psicología, 24(1), 47-60. Recuperado de https://doi.org/10.15446/ rcp.v24n 1.43437 
McGrady, A., Brennan, J., Lynch, D. y Whearty, K. (2012). A wellness program for first year medical students. Applied Psychophysiology and Biofeedback, 37(4), 253-260. Recuperado de https://doi.org/10.1007/ s10484-012-9198-x

Ministerio de Educación Nacional de Colombia. (2009). Deserción estudiantil en la educación superior colombiana: metodología de seguimiento, diagnóstico y elementos para su prevención. Bogotá: Ministerio de Educación Nacional. Recuperado de https://www. mineducacion.gov.co/sistemasdeinformacion/1735/articles-254702_ libro_desercion.pdf

Ostos-Ortiz, O.-L., \& Aparicio-Gómez, O.-Y. Investigación, innovación y transferencia del conocimiento.

Pacheco, M., Michelena, M., Mora, R. y Miranda, O. (2014). Calidad de vida relacionada con la salud en estudiantes universitarios. Revista Cubana de Medicina Militar, 43(2), 157-168. Recuperado de http://scielo. sld.cu/scielo.php?script=sci_arttext\&pid=S0138-65572014000200004

Pezzano, G. (2009). El contexto de la orientación académica desde el bienestar universitario. En E. Llinás (Ed.), La orientación académica desde el bienestar universitario (pp. 1-20). Barranquilla: Ediciones Uninorte. Recuperado de https://www.uninorte.edu.co/documents/72553/ fd13f2c5-352a-4753-9173-5ed18c79c075

Real, F. J., Zackoff, M. W., Davidson, M. A. y Yakes, E. A. (2015). Medical student distress and the impact of a school-sponsored wellness initiative. Medical Science Educator, 25(4), 397-406. Recuperado de https://doi. org/10.1007/s40670-015-0156-0

Rojas, C. (2019). Bienestar universitario y calidad de vida de los estudiantes beneficiarios de la Facultad de Ingeniería - Universidad Nacional de Trujillo, 2019 [tesis de pregrado]. Universidad Nacional de Trujillo, Perú. Recuperado de http://dspace.unitru.edu.pe/ bitstream/handle/UNITRU/15748/Rojas\%20Terrones\%2c\%20Claudia. pdf? sequence $=1 \&$ isAllowed $=y$ 
Runyan, C., Savageau, J. A., Potts, S. y Weinreb, L. (2016). Impact of a family medicine resident wellness curriculum: a feasibility study. Medical Education Online, 21(1), 1-5. Recuperado de https://doi.org/10.3402/ meo.v21.30648

Saadat, H., Snow, D. L., Ottenheimer, S., Dai, F. y Kain, Z. N. (2012). Wellness program for anesthesiology residents: a randomized, controlled trial. Acta Anaesthesiologica Scandinavica, 56(9), 1130-1138. Recuperado de https://doi.org/10.1111/j.1399-6576.2012.02705.x

Salanova, M., Martínez, I., Bresó, E., Llorens, S. y Grau, R. (2005). Bienestar psicológico en estudiantes universitarios: facilitadores y obstaculizadores del desempeño académico. Anales de Psicología, 21(1), 170-180. Recuperado de https://www.um.es/analesps/v21/v21_1/1621_1.pdf

Salcedo, V., Quezada, C., Novillo, E., Varela, G., Núñez, L. y Viteri, C. (2017). Bienestar estudiantil universitario en Ecuador: caso unidades de bienestar estudiantil en las universidades de la provincia de El Oro. Revista Espacios, 38(30), 17-37. Recuperado de http://revistaespacios. com/a17v38n30/17383017.html

Vilaplana, C. (2010). Relación entre los Programas Universitarios para Mayores, la satisfacción durante la jubilación y la calidad de vida. Revista de Investigación Educativa, 28(1), 195-216. Recuperado de https:// revistas.um.es/rie/article/view/97891/109431

Wahyun, E., Nurihsan, J. y Yusuf, S. (2019). Effectiveness of acceptance and commitment therapy to enhance students' wellness. Journal of Evidence-Based Psychotherapies, 19(1), 91-113. Recuperado de https:// doi.org/10.24193/jebp.2019.1.6

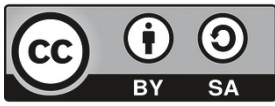

